\title{
36 Simulation as a method of investigation and training
}

The term simulation as employed in this book means: the step-by-step imitation of an inventory and/or production pattern such as might be encountered in reality.

Simulation can be used as a method of exploratory calculation. In certain situations where no effective analytical method is available for calculating the optimum combination of values of $s$ and $Q$, this optimum can be established by simulation.

Another form of investigation worth mentioning is: simulation as applied to the phase 'testing' of a project to ascertain whether a predetermined solution will produce satisfactory results. Alternatively simulation can also be used as a training technique, in which case the decisions are taken by a person without the aid of fixed rules, instead of by a computer. This is what is called an inventory game or an ordering game, the two constituting a special category of business game (see for instance Greene and Sisson [Gr 1], Prins [Pr 1] (1966)).

How the games used in training are actually played depends on whether the training is intended to be general (for instance a general planning course) or specific, as part of the programme for introducing a given ordering system in a particular company.

Space does not permit us to go any further into the different methods of simulation, which is already beginning to emerge as a subject in itself with its own specialized literature; see for instance Tocher [To 1] and Kosten [Ko 1] (1963). 